\title{
AVALIAÇÃO DA EFICIÊNCIA DAS COMPANHIAS AÉREAS BRASILEIRAS UTILIZANDO UM MODELO DEA EM REDE BASEADO EM FOLGAS
}

\author{
Ana Paula dos Santos Rubem \\ Centro de Análise de Sistemas Navais \\ Praça Barão de Ladário s/n, Ilha das Cobras, Centro, 20091-000, Rio de Janeiro, Brasil \\ anarubem@bol.com.br, +552126295450 \\ Plácido Moreno Beltrán \\ Universidad de Sevilla \\ Escuela de Ingenieros, Camino de los Descubrimientos s/n, 4, 41092, Sevilla, España \\ placidomb@us.es, +34616053608 \\ João Carlos Correia Baptista Soares de Mello \\ Universidade Federal Fluminense \\ Rua Passo da Pátria 156, São Domingos, 24210-240, Niterói, RJ, Brasil \\ jcsmello@producao.uff.br, +552126295450
}

\begin{abstract}
RESUMO
Este artigo tem o objetivo de analisar o desempenho operacional de companhias aéreas brasileiras, no período de 2007 a 2010, usando um modelo de Análise Envoltória de Dados (DEA, do inglês Data Envelopment Analysis) em rede. O modelo DEA em rede define cada unidade produtiva como uma rede de estágios, proporcionando um maior entendimento sobre as causas da ineficiência, bem como a decomposição da eficiência global em pontuações individuais para cada estágio. Ao invés de basear o modelo em uma medida radial de eficiência, aplica-se uma métrica baseada em folgas, e assume-se que cada estágio opere sob retornos variáveis de escala, já que os efeitos de escala não podem ser desconsiderados. A análise resultou em nove companhias globalmente eficientes. A divisão do processo produtivo em estágios tornou mais fácil identificar as fontes de ineficiência. Por exemplo, em relação às companhias aéreas ineficientes, seis foram eficientes em pelo menos um estágio.
\end{abstract}

Palavras-chave: Análise Envoltória de Dados. Eficiência. Transporte aéreo.

Área Principal: DEA - Análise Envoltória de Dados.

\section{ABSTRACT}

This article aims at analysing the operational performance of Brazilian airlines from 2007 to 2010, by using a three-stage network Data Envelopment Analysis (DEA) model. Network DEA models define each production unit as a network of stages, providing a greater insight into the causes of inefficiency, as well as the decomposition of the global efficiency into individual scores for each internal stage. Instead of grounding the network DEA model on a radial measure of efficiency, we apply a slacks-based measure metrics. Each stage has been assumed to exhibit variable returns-to-scale, since scale effects cannot be neglected. The analysis resulted in nine globally efficient airlines. The division of the production process in stages made it easier to identify the sources of inefficiency. For instance, regarding the inefficient airlines, six were efficient in at least one stage.

Keywords: Air transport. Data Envelopment Analysis. Efficiency. 


\section{INTRODUÇÃO}

Desde o princípio da década de 1990, o setor aéreo brasileiro vem sofrendo mudanças estruturais significativas, tendo assistido a um processo de desregulamentação, que deu fim à política implantada nos anos 1960, na qual a organização e o comportamento do mercado eram fortemente controlados (Lima et al., 2011). O processo iniciou-se, em 1992, com a extinção dos monopólios regionais, o que incentivou o acesso de novas empresas e a disputa de preços, com a definição de faixas tarifárias. Em 2001, deu-se a liberalização total dos preços, além da maior flexibilidade nos processos de entrada de empresas e pedidos de novas rotas aéreas, frequências de voo e aeronaves, o que levou ao surgimento da Gol, a primeira empresa de baixo custo do país (Evangelho et al., 2005).

Os primeiros sinais de diminuição na competição foram notados por ocasião do code-share entre Varig e TAM (Soares de Mello et al., 2009). A tendência se acentuou em 2006, com as dificuldades enfrentadas pela Varig, que acabou adquirida pela Gol. A partir daí, teve início uma fase de concentração no setor, que levou à redução progressiva na concorrência e à instalação de um quase-duopólio. Todavia, nos últimos três anos, a participação da Azul vem crescendo consideravelmente.

Há vários estudos de aplicação da Análise Envoltória de Dados (DEA, do inglês Data Envelopment Analysis) para avaliar a eficiência de empresas aéreas brasileiras. Boa parte utilizou modelos DEA clássicos, em especial o BCC (Banker et al., 1984), dada a expressiva diferença entre os tamanhos das empresas, que não permite que os efeitos de escala sejam descartados, inibindo o uso do modelo CCR (Charnes et al., 1978) clássico. O BCC assume a hipótese de retornos variáveis de escala (VRS, do inglês Variable Returns-to-Scale), já o CCR a de retornos constantes de escala (CRS, do inglês Constant Returns-to-Scale).

Uma desvantagem dos modelos DEA clássicos reside em negligenciar a estrutura interna das unidades produtivas, tratando-as como uma "caixa preta". Já o DEA em rede (NDEA, do inglês Network Data Envelopment Analysis) define cada unidade produtiva como uma rede de estágios internos interconectados. Portanto, os modelos NDEA permitem revelar as fontes de ineficiência, bem como o cálculo das eficiências nos diferentes nós, decompondo a eficiência global em medidas afetas a cada estágio. Neles, cada estágio pode ter diferentes entradas e saídas exógenas, além de estarem interconectados por produtos intermediários que não são exógenos.

Apesar de os primeiros modelos NDEA serem baseados em medidas radiais de eficiência (e.g., Kao, 2009; Chen et al., 2009), Tone e Tsutsui (2009) estenderam a métrica SBM (do inglês Slacks-Based Measure: Tone, 2001) para uma estrutura em rede aditiva, introduzindo o SBM-NDEA. A métrica SBM é uma medida não-radial de eficiência, que revela as folgas das entradas e saídas.

O objetivo deste trabalho é analisar o desempenho operacional das companhias aéreas brasileiras entre 2007 e 2010. Para tanto, será utilizado um modelo SBM-NDEA aditivo de três estágios, orientado a insumos, sob a suposição VRS, uma vez que as empresas aéreas brasileiras não operam em escalas similares. O modelo engloba três variáveis de entrada, dois produtos intermediários e duas saídas. Devido à orientação a insumos, assume-se que as atividades de ligação também tenham por objetivo a minimização dos produtos intermediários, mantida a continuidade entre as entradas e saídas intermediárias.

A Seção 2 traz uma revisão do uso de DEA no setor aéreo; a Seção 3 descreve o modelo NDEA e a métrica SBM; a Seção 4 apresenta a formulação geral do modelo SBMNDEA; a Seção 5 exibe o modelo de três estágios aplicado; a Seção 6 contém os resultados e uma breve discussão; por fim, as conclusões e sugestões para trabalhos futuros constam da Seção 7.

\section{REVISÃO DE LITERATURA}


A partir da década de 1990, os modelos DEA (Charnes et al., 1978) passaram a ser bastante usados na avaliação da eficiência do setor aéreo. Grande parte dessas aplicações destinou-se à avaliação do desempenho aeroportuário (e.g., Gillen e Lall, 1997; Sarkis, 2000; Adler e Berechman, 2001; Barros e Dieke, 2007; Chi-Lok e Zhang, 2009; Suzuki et al., 2012; Chang et al., 2013; Ha et al., 2013). No caso de aeroportos brasileiros, destacam-se os trabalhos de Fonseca et al. (2010) e Pacheco et al. (2006).

No que se refere à avaliação de empresas aéreas, Schefczyk (1993) utilizou DEA para avaliar o desempenho operacional das companhias, concluindo o estudo com uma análise dos fatores estratégicos ligados à rentabilidade e ao desempenho no setor. Outro exemplo de abordagem DEA não-financeira pode ser visto em Charnes et al. (1996). Adler e Golany (2001) usaram um modelo DEA que cobre aspectos financeiros e não-financeiros. Estudos DEA que avaliam a eficiência da estrutura de capital de empresas brasileiras podem ser vistos em Capobianco e Fernandes (2004) e Fernandes et al. (2008). Com foco em aspectos operacionais, Soares de Mello et al. (2003) aplicaram modelos DEA clássicos para o cálculo da eficiência das companhias aéreas brasileiras de transporte de passageiros. Com propósito similar, Araújo Junior et al. (2007) utilizaram um modelo DEA com ênfase aos principais fatores de produção; enquanto Correia et al. (2011) usaram uma variação do modelo DEA Difuso. A identificação de benchmarks e anti-benchmarks usando um modelo DEA que considera transporte de carga e passageiro, frota e pessoal foi feita por Silveira et al. (2012b). Com as mesmas variáveis, Silveira et al. (2012a) adaptaram o MCDEA para retornos variáveis de escala.

Os estudos acima ignoram a estrutura interna do processo produtivo no cômputo das eficiências. Contudo, desenvolvimentos recentes em DEA (Kao, 2009; Chen et al., 2009; Tone e Tsutsui, 2009) permitiram os estágios internos passassem a ser considerados na avaliação do setor aéreo. No caso de aeroportos, Yu (2010) utilizou um modelo SBM-NDEA para avaliar o desempenho aeroportuário; enquanto Lozano et al. (2013) aplicaram uma abordagem NDEA de distância direcional que permitiu considerar saídas indesejáveis. Com foco na avaliação dos aeroportos brasileiros, Wanke (2013) propôs uma abordagem NDEA de dois estágios. Sobre a avaliação de desempenho operacional de rotas aéreas, Yu (2012) aplicou uma versão modificada do modelo NDEA radial para o planejamento de rota doméstica em Taiwan; ao passo que Yu e Chen (2011) usaram um modelo NDEA fracionário para analisar o desempenho das rotas domésticas de uma companhia em Taiwan. Visando a avaliação do desempenho de empresas aéreas, Zhu et al. (2011) aplicaram um modelo NDEA de dois estágios, com o objetivo de minimizar custos e uso de combustível, dado o load factor e o tamanho da frota. Chang e Yu (2012) realizaram uma análise do desempenho das empresas aéreas internacionais de baixo custo, por meio de uma abordagem SBM-NDEA baseada em estágios de produção e consumo. Finalmente, Lu et al. (2012) aplicaram um modelo NDEA aditivo para avaliar a eficiência de produção e comercialização de empresas aéreas que operam nos Estados Unidos.

Além de representar a primeira avaliação de desempenho operacional das companhias aéreas brasileiras usando um modelo NDEA, outro aspecto inovador deste artigo é o uso da métrica SBM em três estágios: um operacional e dois relativos ao uso da capacidade instalada, sendo um afeto ao transporte de passageiros e outro ao de carga.

\section{NDEA E A MÉTRICA BASEADA EM FOLGAS}

Os modelos DEA, em geral, têm se mostrado apropriados para a análise de eficiência de unidades de produtivas que utilizam múltiplos insumos e múltiplas saídas. Essas unidades produtivas são conhecidas como DMUs (do inglês Decision Making Units). A eficiência é calculada para cada DMU, comparando seus níveis de entradas e saídas com os das outras. O resultado é a construção de uma superfície em forma de envelope ou fronteira eficiente. Apenas as DMUs situadas na fronteira são consideradas eficientes. 
Uma limitação dos modelos DEA usuais consiste em negligenciar os estágios internos dentro de cada DMU e as atividades de ligação entre eles. Nos modelos NDEA, a interligação entre as atividades é uma característica indispensável. Os modelos NDEA com múltiplos estágios em série são os mais comuns. Dada a interconexão estrutural dos modelos NDEA, para que uma DMU possa ser eficiente ela deve ser eficiente em todos os estágios; assim, pode não haver DMUs globalmente eficientes. Nesse tipo de abordagem, os produtos intermediários são produzidos e consumidos na própria DMU, enquanto as entradas e saídas são exógenas a sua estrutura interna. Além da pontuação global, o modelo NDEA permite o cálculo de índices de eficiência para cada estágio. Outra característica é que o conjunto de possibilidades de produção (PPS, do inglês Production Possibility Set) é modelado no nível de cada estágio, que possui seu próprio nível tecnológico, com coeficientes de referência específicos.

O modelo NDEA foi desenvolvido por Färe e Grosskopf (2000), embora os primeiros trabalhos ainda desprezassem a continuidade entre os nós, resolvendo um modelo DEA independente para cada estágio. Kao (2009) incorporou a interconexão entre os estágios com a abordagem NDEA-CRS relacional (ou multiplicativa); Chen et al. (2009) propuseram um modelo NDEA-VRS aditivo, que também considera a continuidade dos produtos intermediários; enquanto Tone e Tsutsui (2009) adaptaram as métricas SBM ao modelo NDEA-VRS aditivo, de modo que as ineficiências não radiais pudessem ser detectadas. No modelo multiplicativo a eficiência global é o produto das eficiências de cada estágio, enquanto no aditivo é a média ponderada das eficiências de cada estágio. Neste estudo, tal ponderação é feita com base na quantidade de variáveis de entrada (exógenas e intermediárias) de cada estágio.

Introduzida por Tone (2001), a abordagem SBM é uma métrica não-radial para avaliar a eficiência, que lida diretamente com os excessos de entrada e os déficits de saída (folgas) da DMU em análise. Enquanto as abordagens radiais proporcionam a redução equiproporcional dos insumos ou o aumento equiproporcional dos produtos determinados pelas projeções radiais, a métrica SBM permite a variação não-equiproporcional de entradas e saídas ao buscar alvos para as DMUs ineficientes.

Nos modelos SBM-NDEA, sob a condição VRS, cada estágio tem ao menos uma DMU eficiente; ao passo que, sob a hipótese CRS, é possível que algum estágio não tenha DMU eficiente (Tone e Tsutsui, 2009). Logo, pode não haver uma DMU globalmente eficiente, diferentemente dos modelos DEA usuais. Aqui, será aplicado um modelo SBMNDEA aditivo de três estágios, sob a hipótese VRS, pois é inadequado supor que as empresas aéreas brasileiras operam em escalas similares.

\section{FORMULAÇÃO GERAL DO MODELO}

Suponha n DMUs $(\mathrm{j}=1, \ldots, \mathrm{n})$, cada uma conduzindo um processo produtivo com $\mathrm{K}$ $(\mathrm{k}=1, \ldots, \mathrm{K})$ estágios. Seja $\mathrm{m}_{\mathrm{k}}$ e $\mathrm{r}_{\mathrm{k}} \mathrm{o}$ número de entradas e saídas do estágio $\mathrm{k}$, respectivamente. Seja o elo do estágio k ao estágio h denotado por $(\mathrm{k}, \mathrm{h})$ e o conjunto de elos por L. Os dados observados são:

$\left\{\mathbf{x}_{\mathrm{j}}^{\mathrm{k}} \in \mathrm{R}_{+}^{\mathrm{m}_{\mathrm{k}}}\right\},(\mathrm{j}=1, \ldots, \mathrm{n} ; \mathrm{k}=1, \ldots, \mathrm{K})$ - entradas exógenas da $\mathrm{DMU} \mathrm{U}_{\mathrm{j}}$ no estágio $\mathrm{k}$;

$\left\{y_{j}^{k} \in R_{+}^{r_{k}}\right\},(j=1, \ldots, n ; k=1, \ldots, K)$ - saídas finais da $D M U_{j}$ no estágio $k$; e

$\left\{\mathbf{z}_{\mathrm{j}}^{(\mathrm{k}, \mathrm{h})} \in \mathrm{R}_{+}^{\mathrm{t}(\mathrm{k}, \mathrm{h})}\right\},(\mathrm{j}=1, \ldots, \mathrm{n} ;(\mathrm{k}, \mathrm{h}) \in \mathrm{L})$ - produtos intermediários da $\mathrm{DMU}_{\mathrm{j}}$ do estágio $\mathrm{k}$ para o estágio $h$, onde $t_{(k, h)}$ é o número de produtos no elo $(k, h)$.

O PPS $\left\{\left(\mathbf{X}^{\mathrm{k}}, \mathbf{Y}^{\mathrm{k}}, \mathbf{Z}^{(\mathrm{k}, \mathrm{h})}\right)\right\}$ é definido por:

$\mathbf{X}^{\mathrm{k}} \geq \sum_{\mathrm{j}=1}^{\mathrm{n}} \mathbf{x}_{\mathrm{j}}^{\mathrm{k}} \lambda_{\mathrm{j}}^{\mathrm{k}}, \quad(\mathrm{k}=1, \ldots, \mathrm{K})$;

$\mathbf{Y}^{\mathrm{k}} \leq \sum_{\mathrm{j}=1}^{\mathrm{n}} \mathbf{y}_{\mathrm{j}}^{\mathrm{k}} \lambda_{\mathrm{j}}^{\mathrm{k}}, \quad(\mathrm{k}=1, \ldots, \mathrm{K}) ;$

$\mathbf{Z}^{(\mathrm{k}, \mathrm{h})}=\sum_{\mathrm{j}=1}^{\mathrm{n}} \mathbf{z}_{\mathrm{j}}^{(\mathrm{k}, \mathrm{h})} \lambda_{\mathrm{j}}^{\mathrm{k}}, \forall(\mathrm{k}, \mathrm{h})$ (saídas do estágio k); 
$\mathbf{Z}^{(\mathrm{k}, \mathrm{h})}=\sum_{\mathrm{j}=1}^{\mathrm{n}} \mathbf{z}_{\mathrm{j}}^{(\mathrm{k}, \mathrm{h})} \lambda_{\mathrm{j}}^{\mathrm{h}}, \quad \forall(\mathrm{k}, \mathrm{h})$ (entradas do estágio $\mathrm{h}$ );

$\sum_{\mathrm{j}=1}^{\mathrm{n}} \lambda_{\mathrm{j}}^{\mathrm{k}}=1, \forall \mathrm{k}$

$\lambda_{\mathrm{j}}^{\mathrm{k}} \geq 0, \forall(\mathrm{j}, \mathrm{k})$;

onde $\lambda^{\mathrm{k}} \in \mathrm{R}_{+}^{\mathrm{n}}$ é o conjunto de vetores de intensidade do estágio $\mathrm{k}$.

O PPS acima assume a hipótese VRS para a produção. Como a abordagem é orientada a insumos para toda a rede de processos, faz sentido organizar os produtos intermediários de modo a serem minimizados, i.e., tratando-os como entradas. Os excessos no valor dos produtos intermediários contarão como ineficiências para o estágio em que o produto intermediário é uma entrada. Além disso, vão haver restrições para garantir a continuidade de produtos intermediários entre as fases consecutivas (Tone e Tsutsui, 2009). A eficiência global, orientada a insumos, da $\operatorname{DMU}_{\mathrm{o}}(\mathrm{o}=1, \ldots, \mathrm{n})$ é avaliada por meio da resolução do seguinte programa linear:

$$
\theta_{\mathrm{o}}^{*}=\min \left[1-\frac{1}{\sum_{\mathrm{k}=1}^{\mathrm{K}}\left(\mathrm{m}_{\mathrm{k}}+\sum_{\mathrm{f} \in \mathrm{P}_{\mathrm{k}}} \mathrm{t}_{(\mathrm{f}, \mathrm{k})}\right)} \sum_{\mathrm{k}=1}^{\mathrm{K}}\left(\sum_{\mathrm{i}=1}^{\mathrm{m}_{\mathrm{k}}} \frac{\mathrm{s}_{\mathrm{io}}^{\mathrm{k}-}}{\mathrm{x}_{\mathrm{io}}^{\mathrm{k}}}+\sum_{\mathrm{f} \in \mathrm{P}_{\mathrm{k}}} \sum_{\mathrm{l}=1}^{\mathrm{t}_{(\mathrm{f}, \mathrm{k})}} \frac{\mathrm{s}_{\mathrm{lo}}^{(\mathrm{f}, \mathrm{k})-}}{\mathrm{z}_{\mathrm{lo}}^{(\mathrm{f}, \mathrm{k})}}\right)\right],
$$

sujeito a:

$\mathbf{x}_{\mathrm{o}}^{\mathrm{k}}=\mathbf{X}^{\mathrm{k}} \lambda^{\mathrm{k}}+\mathbf{s}_{\mathbf{o}}^{\mathrm{k}-}, \quad(\mathrm{k}=1, \ldots, \mathrm{K}) ;$

$\mathbf{y}_{0}^{\mathrm{k}} \leq \mathbf{Y}^{\mathrm{k}} \lambda^{\mathrm{k}}, \quad(\mathrm{k}=1, \ldots, \mathrm{K})$;

$\mathbf{e} \lambda^{\mathrm{k}}=1, \quad(\mathrm{k}=1, \ldots, \mathrm{K})$;

$\lambda^{\mathrm{k}} \geq \mathbf{0}, \mathbf{s}_{\mathbf{0}}^{\mathrm{k}-} \geq \mathbf{0}, \forall \mathrm{k}$

$\mathbf{z}_{\mathrm{lo}}^{(\mathrm{f}, \mathrm{k})}=\mathbf{Z}^{(\mathrm{f}, \mathrm{k})} \lambda^{\mathrm{k}}+\mathbf{s}_{\mathrm{lo}}^{(\mathrm{f}, \mathrm{k})-}, \quad \forall(\mathrm{f}, \mathrm{k})$;

$\mathbf{Z}^{(\mathrm{f}, \mathrm{k})} \boldsymbol{\lambda}^{\mathrm{f}}=\mathbf{Z}^{(\mathrm{f}, \mathrm{k})} \boldsymbol{\lambda}^{\mathrm{k}}, \forall(\mathrm{f}, \mathrm{k})$;

$\mathbf{s}_{\mathrm{lo}}^{(\mathrm{f}, \mathrm{k})-} \geq \mathbf{0}, \forall(\mathrm{f}, \mathrm{k})$;

onde $\quad \mathbf{X}^{\mathrm{k}}=\left(\mathbf{x}_{1}^{\mathrm{k}}, \ldots, \mathbf{x}_{\mathrm{n}}^{\mathrm{k}}\right) \in \mathrm{R}_{+}^{\mathrm{m}_{\mathrm{k}} \times \mathrm{n}}, \mathbf{Y}^{\mathrm{k}}=\left(\mathbf{y}_{1}^{\mathrm{k}}, \ldots, \mathbf{y}_{\mathrm{n}}^{\mathrm{k}}\right) \in \mathrm{R}_{+}^{\mathrm{r}_{\mathrm{k}} \times \mathrm{n}}, \mathbf{Z}^{\mathrm{k}}=\left(\mathbf{z}_{1}^{(\mathrm{k}, \mathrm{h})}, \ldots, \mathbf{z}_{\mathrm{n}}^{(\mathrm{k}, \mathrm{h})}\right) \in$ $\mathrm{R}_{+}^{\mathrm{t}(\mathrm{k}, \mathrm{h})} \times \mathrm{n} ; \mathbf{s}_{\mathbf{o}}^{\mathrm{k}-}$ são os vetores de folga das entradas (saídas); $\mathbf{s}_{\mathbf{l o}}^{(\mathrm{f}, \mathrm{k})-}$ é o vetor de folga dos elos (f, $\mathrm{k})$ como entrada do estágio $\mathrm{k}$; $\mathrm{P}_{\mathrm{k}}$ é o conjunto de estágios com elo (f, $\left.\mathrm{k}\right) \in \mathrm{L}$ para um dado estágio $\mathrm{k}$; e $\mathrm{t}_{(\mathrm{f}, \mathrm{k})}$ é o número de itens naquele elo.

Por definição, uma DMU é globalmente eficiente se $\theta_{0}^{*}=1$. Isso equivale a todas as folgas serem nulas, uma vez que as folgas correspondem aos excessos de entradas ou de produtos intermediários que impedem que a unidade seja eficiente. O conjunto de vetores de intensidade positivos $\left(\lambda^{\mathrm{k}}\right)$ é o conjunto de referência de unidades eficientes que estabelecem os alvos para a DMU em avaliação. Como já mencionado, NDEA envolve um conjunto de referências (benchmarks) diferente para cada estágio k. Uma vez resolvido o programa linear acima e calculados os valores ideais para o conjunto de folgas e os vetores de intensidade, as eficiências dos K estágios são dadas pela fórmula abaixo. A DMU é eficiente no estágio k, se $\theta_{\mathrm{o}}^{* \mathrm{k}}=1$. Uma DMU é globalmente eficiente somente se ela é eficiente em todos os estágios.

$$
\theta_{\mathrm{o}}^{* \mathrm{k}}=\min \left[1-\frac{1}{\left(\mathrm{~m}_{\mathrm{k}}+\sum_{\mathrm{f} \in \mathrm{P}_{\mathrm{k}}} \mathrm{t}_{(\mathrm{f}, \mathrm{k})}\right)}\left(\sum_{\mathrm{i}=1}^{\mathrm{m}_{\mathrm{k}}} \frac{\mathrm{s}_{\mathrm{io}}^{* \mathrm{k}-}}{\mathrm{x}_{\mathrm{io}}^{\mathrm{k}}}+\sum_{\mathrm{f} \in \mathrm{P}_{\mathrm{k}}} \sum_{\mathrm{l}=1}^{\mathrm{t}_{\mathrm{f}, \mathrm{k})}} \frac{\mathrm{s}_{\mathrm{lo}}^{*(\mathrm{f}, \mathrm{k})-}}{\mathrm{z}_{\mathrm{lo}}^{(\mathrm{f}, \mathrm{k})}}\right)\right]
$$

\section{MODELAGEM}

Com ênfase nos principais fatores de produção (capital, energia e mão de obra), este estudo tem o objetivo de avaliar o desempenho operacional das empresas aéreas brasileiras, 
usando um modelo que leva em conta o transporte de passageiros e de carga, frota, custos de combustível e pessoal, tanto em voos nacionais como internacionais.

Para tanto, será utilizado um modelo SBM-NDEA aditivo de três estágios, orientado a insumos, sob a hipótese VRS, em todos os estágios. O modelo considera três variáveis exógenas de entrada (custo de combustível, pessoal total e capacidade da frota), dois produtos intermediários (assentos.km e toneladas.km oferecidas) e duas saídas finais (passageiros.km e toneladas.km transportadas). Adotou-se uma configuração híbrida para o processo produtivo, em série no primeiro e paralela nos segundo e terceiro estágios, já que carga e passageiros compreendem subprocessos distintos. A estrutura utilizada está ilustrada na Figura 1.

O primeiro estágio está associado à capacidade da empresa de colocar aeronaves no ar com os recursos disponíveis. As entradas são os recursos utilizados para a operação do serviço. A otimização da frota (principal bem de capital das empresas) evita a ociosidade das aeronaves, um dos fatores de produção que contribui para a redução dos custos operacionais e, portanto, para o aumento dos lucros da empresa. Os outros fatores de produção englobados no primeiro estágio do modelo são custos de combustível (energia) e pessoal total (mão de obra), como considerado por Soares de Mello et al. (2003) em estudo anterior sobre a eficiência das companhias aéreas brasileiras (abordagem similar pode ser vista em Araújo Junior et al., 2007).

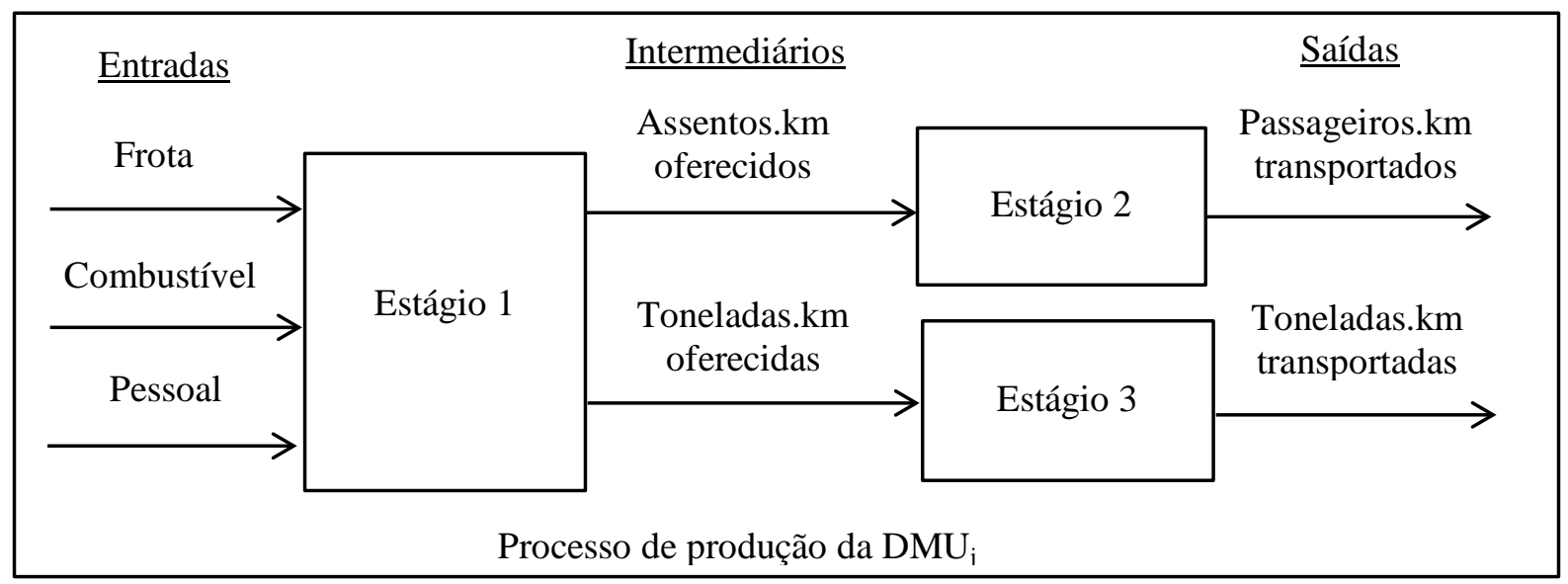

Figura 1 - Representação de um sistema misto de três estágios.

Como cada empresa usa modelos distintos, com capacidade diferenciada, a entrada "capacidade da frota", tal como em Correia et al. (2011), indica a soma dos pesos máximos de decolagem de todas as aeronaves pertencentes à empresa no ano em questão; englobando, simultaneamente, o total de aviões e as respectivas capacidades. A entrada "pessoal total" inclui pessoal de voo, técnico, de tráfego, de vendas e outros, denotando o total de pessoas empregadas pela empresa. Já a entrada "custo de combustível" representa o custo direto de combustível em R\$, corrigido até 2010, pelo índice Nacional de Preços ao Consumidor Amplo.

O serviço oferecido por cada companhia é o transporte de passageiros e carga. Logo, o segundo e o terceiro estágios estão associados à utilização da capacidade instalada das aeronaves (load factors) na prestação do serviço de transporte de passageiros e carga, respectivamente. Como os resultados finais do modelo devem estar relacionados ao serviço oferecido, as saídas consideradas são o número de passageiros transportados multiplicado pelos quilômetros voados (passageiros.km transportados) e as toneladas transportadas multiplicadas pelos quilômetros voados (toneladas.km transportadas).

Já os produtos intermediários são o total de assentos oferecidos multiplicado pelos quilômetros voados (assentos.km oferecidos) e as toneladas oferecidas multiplicadas pelos quilômetros voados (toneladas.km oferecidas). A variável intermediária "assentos.km oferecidos" é tanto uma saída do estágio 1 como uma entrada do estágio 2; enquanto a 
variável "toneladas.km oferecidas" é, ao mesmo tempo, uma saída do estágio 1 e entrada do estágio 3.

Trabalhos anteriores sobre eficiência NDEA de companhias aéreas (e.g., Lu et al. 2012), além de usarem uma abordagem radial, consideravam uma estrutura de apenas dois estágios, englobando as eficiências de produção e comercialização, respectivamente, similares ao estágio 1 e à combinação dos estágios 2 e 3 aqui propostos. Acredita-se, porém, que a divisão do estágio de comercialização em duas etapas paralelas poderá propiciar uma análise mais acurada, permitindo, por exemplo, que se avalie o impacto do emprego de políticas diferenciadas quanto ao transporte de passageiros e carga na eficiência revelada pelas empresas.

A hipótese VRS foi assumida, pois as empresas analisadas operam em escalas bem diferenciadas; além disso, utilizou-se uma orientação a insumos para identificar as que podem reduzir, ao menos, um dos seus recursos (exógenos e/ou intermediários). Como foi usada uma abordagem não-radial, é permitido o aumento ou decréscimo simultâneo nas saídas (finais e/ou intermediárias). A principal vantagem do SBM-NDEA em relação às abordagens radiais é a possibilidade de definir alvos para todas as variáveis envolvidas, mesmo as intermediárias.

O modelo foi aplicado ao período de 2007 a 2010. As observações foram incluídas em conjunto na análise, uma vez que a regulação e estrutura permaneceu praticamente inalterada durante o período. Os dados foram retirados dos Anuários Estatísticos de Transporte Aéreo, disponíveis no site da Agência Nacional de Aviação Civil (ANAC, 2007, 2008, 2009, 2010). As DMU são as empresas aéreas regulares de transporte de carga e passageiros, considerando-se a mesma empresa como uma DMU distinta em anos diferentes. Essa é uma das formas de se aumentar o número de DMUs, sugerida por Podinovski e Thanassoulis (2007), que possibilita, ainda, a análise temporal, tal como em Soares de Mello et al. (2003), dentre outros.

A análise começa em 2007, ano seguinte à crise no setor aéreo brasileiro, já que optou-se por estudar apenas anos com características similares, para que houvesse maior consistência nos dados. Os dados de 2011 e 2012, embora já estejam disponíveis na página da ANAC, não contêm todas as variáveis consideradas no modelo, por isso a análise se encerra em 2010.

Devido à agregação de passageiros e carga em quilômetros transportados no ano, os dados referem-se à posição de dezembro. Da mesma forma, os dados da frota, embora se saiba que possam variar durante o ano. Assim, para estimar a posição da frota no fim do primeiro semestre, foram calculadas a média aritmética simples, incorporando o ano anterior e o ano em curso; em seguida, a média foi usada para calcular a variável de entrada "capacidade da frota”.

\section{RESULTADOS E DISCUSSÃO}

No período em análise, observou-se uma média anual de 26 empresas aéreas brasileiras operando no transporte de carga e passageiros, que correspondem a 103 DMUs (combinações empresa-ano). Dentre elas, 27 foram descartadas da análise, por operarem estritamente no transporte de carga. Outras 7 DMUs não puderam ser consideradas, pois não havia informação sobre algumas das variáveis de entrada. Assim, a modelagem proposta foi aplicada às 69 DMUs restantes. Esse número satisfaz os resultados empíricos relatados por Banker et al. (1989), segundo os quais para a obtenção de resultados confiáveis a quantidade de DMUs deve ser, pelo menos, igual a três vezes o número total de variáveis de entradas, saídas e intermediárias.

A Tabela 1 exibe os resultados em termos de eficiência global e específicas de cada estágio. Dentre as 69 DMUs analisadas, nove foram classificadas como globalmente eficientes. Como já mencionado, devido à interconexão estrutural do modelo, uma DMU globalmente eficiente deve ser eficiente em todos os estágios. Das unidades produtivas

globalmente eficientes, três referem-se ao ano de 2010 (Rico, Sol e TAM), três a 2009 
(Abaeté, Puma e Sol), duas a 2008 (Abaeté e TAM) e apenas uma ao ano de 2007 (Mega). O fato de o ano de 2007 apresentar o menor número de empresas eficientes, pode ser reflexo da crise um ano antes.

Devido à suposição VRS, há DMUs globalmente eficientes não necessariamente por suas práticas, mas possivelmente apenas por apresentarem o menor (maior) valor para uma entrada ou saída em algum dos estágios (eficiente por default). A TAM, em 2010foi classificada como globalmente eficiente por exibir os maiores valores em pelos menos uma variável de saída (intermediária e final) a cada estágio. Outras quatro DMUs globalmente eficientes, embora não tenham sido eficientes por default em todos os estágios, o foram em ao menos um (Rico, em 2010; Puma e Sol, em 2009; e Mega, 2007). Esclarecimentos adicionais sobre eficiência técnica e de escala em modelos NDEA podem ser obtidos em Lozano (2011).

A decomposição aditiva da eficiência permitiu a descoberta de eficiências não aparentes. Embora não sejam globalmente eficientes, seis DMUs apresentaram ao menos um estágio com pontuação igual a 100\%, nenhuma por default. Mais precisamente, duas DMUs foram eficientes no segundo estágio (Azul e Total, em 2010) e cinco no terceiro (Azul, em 2010; Gol/Varig, em 2009; Puma, em 2008, e Puma e Sete, em 2007). Em 2010, a Azul foi eficiente em dois estágios. Nenhuma das DMUs ineficientes foi eficiente no primeiro estágio, indicando que a eficiência em todo o processo parece estar fortemente relacionada ao desempenho no primeiro estágio.

A decomposição pode ser especialmente útil para as DMUs ineficientes, pois facilita a identificação das fontes de ineficiência, servindo como um guia para as políticas de gestão. Por exemplo, uma companhia ineficiente apenas no primeiro estágio pode reduzir ao menos uma de suas entradas para se tornar eficiente, sem a obrigatoriedade de manter os níveis dos produtos intermediários, já que foi utilizada uma abordagem não-radial, orientada a insumos. Análise semelhante pode ser feita para empresas ineficientes só no segundo ou terceiro estágio. Além disso, quando a empresa é ineficiente em mais de um estágio, quanto menor a pontuação em um estágio específico, mais ele pode contribuir para melhorar o desempenho global. Assim, quanto pior a pontuação em um estágio, mais esforço a empresa deve empreender para elevar o desempenho naquela etapa específica. Por exemplo, em 2008, a empresa Pantanal, apesar de ineficiente em todos os estágios, apresentou pontuação mais baixa no primeiro estágio (28\%). Logo, para melhorar seu desempenho global, ela deve focar, principalmente, no primeiro estágio. Análise similar pode ser conduzida para todas as DMUs ineficientes.

Tabela 1 - Eficiências sob o modelo SBM-NDEA-VRS de três estágios, orientado a insumos.

\begin{tabular}{|c|c|c|c|c|c|c|c|c|c|c|c|}
\hline \multirow[b]{2}{*}{ Ano } & \multirow[b]{2}{*}{ Empresas } & \multicolumn{4}{|c|}{ Eficiências } & \multirow[b]{2}{*}{ Ano } & \multirow[b]{2}{*}{ Empresas } & \multicolumn{4}{|c|}{ Eficiências } \\
\hline & & Global & $\begin{array}{c}\text { Estágio } \\
1 \\
\end{array}$ & $\begin{array}{c}\text { Estágio } \\
2 \\
\end{array}$ & $\begin{array}{c}\text { Estágio } \\
2 \\
\end{array}$ & & & Global & $\begin{array}{c}\text { Estágio } \\
1 \\
\end{array}$ & $\begin{array}{c}\text { Estágio } \\
2 \\
\end{array}$ & $\begin{array}{c}\text { Estágio } \\
2 \\
\end{array}$ \\
\hline \multirow{17}{*}{2010} & Abaete & $70,6 \%$ & $81,4 \%$ & $55,9 \%$ & $52,9 \%$ & \multirow{17}{*}{2008} & Abaete & $100,0 \%$ & $100,0 \%$ & $100,0 \%$ & $100,0 \%$ \\
\hline & Avianca & $66,0 \%$ & $55,2 \%$ & $92,6 \%$ & $71,8 \%$ & & Air Minas & $41,2 \%$ & $23,9 \%$ & $67,1 \%$ & $67,2 \%$ \\
\hline & Azul & $91,7 \%$ & $86,1 \%$ & $100,0 \%$ & $100,0 \%$ & & Gol/Varig & $77,7 \%$ & $71,7 \%$ & $85,6 \%$ & $88,0 \%$ \\
\hline & Gol/Varig & $92,3 \%$ & $91,2 \%$ & $93,0 \%$ & $94,7 \%$ & & NHT & $28,2 \%$ & $21,3 \%$ & $58,4 \%$ & $18,9 \%$ \\
\hline & NHT & $31,4 \%$ & $23,9 \%$ & $41,0 \%$ & $44,4 \%$ & & Oceanair & $48,0 \%$ & $41,9 \%$ & $81,9 \%$ & $32,5 \%$ \\
\hline & Noar & $55,4 \%$ & $57,3 \%$ & $56,5 \%$ & $48,6 \%$ & & Pantanal & $43,7 \%$ & $22,9 \%$ & $67,8 \%$ & $82,1 \%$ \\
\hline & Pantanal & $53,3 \%$ & $46,5 \%$ & $67,9 \%$ & $59,3 \%$ & & Passaredo & $43,5 \%$ & $30,7 \%$ & $64,2 \%$ & $61,1 \%$ \\
\hline & Passaredo & $53,1 \%$ & $39,8 \%$ & $82,3 \%$ & $64,0 \%$ & & Puma & $63,7 \%$ & $45,6 \%$ & $81,6 \%$ & $100,0 \%$ \\
\hline & Puma & $58,0 \%$ & $60,3 \%$ & $51,6 \%$ & $57,8 \%$ & & Rico & $47,5 \%$ & $25,4 \%$ & $80,8 \%$ & $80,5 \%$ \\
\hline & Rico & $100,0 \%$ & $100,0 \%$ & $100,0 \%$ & $100,0 \%$ & & Sete & $43,5 \%$ & $26,9 \%$ & $69,2 \%$ & $67,6 \%$ \\
\hline & Sete & $43,1 \%$ & $26,3 \%$ & $75,6 \%$ & $60,9 \%$ & & TAF & $38,7 \%$ & $16,4 \%$ & $77,0 \%$ & $67,3 \%$ \\
\hline & Sol & $100,0 \%$ & $100,0 \%$ & $100,0 \%$ & $100,0 \%$ & & TAM & $100,0 \%$ & $100,0 \%$ & $100,0 \%$ & $100,0 \%$ \\
\hline & TAM & $100,0 \%$ & $100,0 \%$ & $100,0 \%$ & $100,0 \%$ & & Team & $34,9 \%$ & $30,6 \%$ & $44,1 \%$ & $38,7 \%$ \\
\hline & Team & $37,5 \%$ & $38,1 \%$ & $40,0 \%$ & $33,2 \%$ & & Total & $41,4 \%$ & $9,3 \%$ & $95,5 \%$ & $83,5 \%$ \\
\hline & Total & $45,3 \%$ & $13,9 \%$ & $100,0 \%$ & $84,6 \%$ & & Trip & $54,1 \%$ & $36,7 \%$ & $78,5 \%$ & $82,1 \%$ \\
\hline & Trip & $53,0 \%$ & $43,6 \%$ & $77,8 \%$ & $56,4 \%$ & & Webjet & $65,4 \%$ & $60,8 \%$ & $83,8 \%$ & $61,1 \%$ \\
\hline & Webjet & $93,4 \%$ & $92,6 \%$ & $95,3 \%$ & $93,8 \%$ & & & & & & \\
\hline 2009 & Abaete & $100,0 \%$ & $100,0 \%$ & $100,0 \%$ & $100,0 \%$ & 2007 & Abaete & $56,7 \%$ & $67,4 \%$ & $46,4 \%$ & $35,0 \%$ \\
\hline
\end{tabular}




\begin{tabular}{|l|r|r|r|r||l|l|r|r|r|} 
Air Minas & $39,0 \%$ & $24,9 \%$ & $62,8 \%$ & $57,3 \%$ & Air Minas & $36,1 \%$ & $25,2 \%$ & $47,9 \%$ & $57,0 \%$ \\
Avianca & $59,9 \%$ & $49,3 \%$ & $89,1 \%$ & $62,3 \%$ & Gol & $90,9 \%$ & $90,9 \%$ & $90,9 \%$ & $90,9 \%$ \\
Azul & $92,3 \%$ & $89,8 \%$ & $94,9 \%$ & $97,2 \%$ & Mega & $100,0 \%$ & $100,0 \%$ & $100,0 \%$ & $100,0 \%$ \\
Gol/Varig & $88,5 \%$ & $85,0 \%$ & $87,8 \%$ & $100,0 \%$ & NHT & $29,8 \%$ & $24,8 \%$ & $55,9 \%$ & $18,7 \%$ \\
NHT & $35,9 \%$ & $24,9 \%$ & $50,6 \%$ & $54,3 \%$ & Oceanair & $36,0 \%$ & $27,0 \%$ & $72,7 \%$ & $26,5 \%$ \\
Pantanal & $45,5 \%$ & $30,5 \%$ & $67,9 \%$ & $68,0 \%$ & Pantanal & $46,7 \%$ & $28,0 \%$ & $67,3 \%$ & $82,0 \%$ \\
Passaredo & $57,1 \%$ & $48,5 \%$ & $80,6 \%$ & $59,3 \%$ & Passaredo & $54,3 \%$ & $42,6 \%$ & $73,3 \%$ & $70,6 \%$ \\
Puma & $100,0 \%$ & $100,0 \%$ & $100,0 \%$ & $100,0 \%$ & Puma & $59,7 \%$ & $39,4 \%$ & $80,3 \%$ & $100,0 \%$ \\
Rico & $40,4 \%$ & $26,7 \%$ & $70,6 \%$ & $51,3 \%$ & Rico & $49,7 \%$ & $36,9 \%$ & $81,6 \%$ & $56,2 \%$ \\
Sete & $40,6 \%$ & $27,8 \%$ & $68,7 \%$ & $51,0 \%$ & Sete & $51,0 \%$ & $29,4 \%$ & $67,1 \%$ & $100,0 \%$ \\
Sol & $100,0 \%$ & $100,0 \%$ & $100,0 \%$ & $100,0 \%$ & TAF & $52,5 \%$ & $38,4 \%$ & $70,4 \%$ & $76,9 \%$ \\
TAF & $36,2 \%$ & $19,6 \%$ & $69,1 \%$ & $53,1 \%$ & TAM & $95,3 \%$ & $94,6 \%$ & $97,5 \%$ & $95,1 \%$ \\
TAM & $94,4 \%$ & $95,5 \%$ & $94,7 \%$ & $91,1 \%$ & Team & $31,8 \%$ & $28,2 \%$ & $38,7 \%$ & $35,5 \%$ \\
Team & $38,1 \%$ & $39,4 \%$ & $39,7 \%$ & $32,7 \%$ & Total & $42,4 \%$ & $17,5 \%$ & $77,5 \%$ & $81,8 \%$ \\
Total & $44,9 \%$ & $14,0 \%$ & $100,0 \%$ & $82,3 \%$ & Trip & $48,9 \%$ & $33,4 \%$ & $75,5 \%$ & $69,1 \%$ \\
Trip & $53,0 \%$ & $40,8 \%$ & $75,2 \%$ & $67,5 \%$ & Varig & $50,2 \%$ & $34,8 \%$ & $67,2 \%$ & $79,3 \%$ \\
Webjet & $71,7 \%$ & $72,8 \%$ & $84,0 \%$ & $56,2 \%$ & Webjet & $63,4 \%$ & $52,2 \%$ & $77,9 \%$ & $82,3 \%$ \\
\hline
\end{tabular}

A Tabela 2 mostra a evolução da eficiência global por empresa. Algumas melhoraram seu desempenho geral ano a ano, atuando como seu próprio benchmark (Avianca, Gol/Varig, Oceanair e Webjet). Embora ineficientes, sete DMUs têm índice superior a 90\% (TAM, em 2009 e 2007; Webjet, em 2010; Azul, em 2010 e 2009; Gol/Varig, em 2010, e Gol, em 2007).

Tabela 2 - Evolução temporal das eficiências globais, de 2010 a 2007, por empresa.

\begin{tabular}{|c|c|c|c|c|c|c|c|c|c|}
\hline \multicolumn{5}{|c|}{ Eficiência Global } & \multicolumn{5}{|c|}{ Eficiência Global } \\
\hline \multirow{2}{*}{ Empresa } & \multicolumn{4}{|c|}{ Ano } & \multirow{2}{*}{ Empresa } & \multicolumn{4}{|c|}{ Ano } \\
\hline & 2010 & 2009 & 2008 & 2007 & & 2010 & 2009 & 2008 & 2007 \\
\hline Abaete & 70,61\% & $100,00 \%$ & $100,00 \%$ & $56,71 \%$ & Puma & $58,04 \%$ & $100,00 \%$ & $63,68 \%$ & $59,72 \%$ \\
\hline Air Minas & - & $38,96 \%$ & $41,17 \%$ & $36,13 \%$ & Rico & $100,00 \%$ & $40,42 \%$ & $47,51 \%$ & $49,68 \%$ \\
\hline Avianca & $65,96 \%$ & $59,89 \%$ & - & - & Sete & $43,10 \%$ & $40,61 \%$ & $43,50 \%$ & $51,05 \%$ \\
\hline Azul & $91,67 \%$ & $92,33 \%$ & - & - & Sol & $100,00 \%$ & $100,00 \%$ & - & - \\
\hline Gol & - & - & - & $90,94 \%$ & TAF & - & $36,18 \%$ & - & $52,53 \%$ \\
\hline Gol/Varig & $92,28 \%$ & $88,55 \%$ & 77,73\% & - & TAM & $100,00 \%$ & $94,44 \%$ & $100,00 \%$ & $95,28 \%$ \\
\hline Mega & - & - & - & $100,00 \%$ & Team & $37,49 \%$ & $38,14 \%$ & $34,94 \%$ & $31,77 \%$ \\
\hline NHT & $31,43 \%$ & $35,92 \%$ & $28,23 \%$ & $29,79 \%$ & Total & $45,29 \%$ & $44,85 \%$ & $41,40 \%$ & $42,36 \%$ \\
\hline Noar & $55,42 \%$ & - & - & - & Trip & $52,99 \%$ & $53,05 \%$ & $54,13 \%$ & $48,93 \%$ \\
\hline Oceanair & - & - & $48,03 \%$ & $36,01 \%$ & Varig & - & - & - & $50,18 \%$ \\
\hline Pantanal & $53,32 \%$ & $45,47 \%$ & $43,74 \%$ & $46,66 \%$ & & & & & $63,36 \%$ \\
\hline Passaredo & $53,13 \%$ & $57,08 \%$ & $43,50 \%$ & 54,33 & & & & & \\
\hline
\end{tabular}

A Tabela 3 relaciona as eficiências médias globais e específicas de cada estágio, para cada ano. A eficiência global média cresceu desde 2007, indicando que as empresas tornaramse mais homogêneas desde então. As eficiências médias do primeiro estágio apresentaram os maiores acréscimos (de cerca de 45\%, em 2007, para mais de 62\%, em 2010). Portanto, o aumento da eficiência média global se deve, principalmente, a melhorias no primeiro estágio.

Tabela 3 - Média das eficiências globais e de cada estágio, de 2010 a 2007.

\begin{tabular}{|c|c|c|c|c|}
\hline \multirow{2}{*}{ Ano } & \multicolumn{4}{|c|}{ Eficiências Médias } \\
\cline { 2 - 5 } & Global & Estágio 1 & Estágio 2 & Estágio 3 \\
\hline 2010 & $67,30 \%$ & $62,13 \%$ & $78,20 \%$ & $71,91 \%$ \\
2009 & $63,20 \%$ & $54,97 \%$ & $79,76 \%$ & $71,31 \%$ \\
2008 & $54,48 \%$ & $41,50 \%$ & $77,22 \%$ & $70,68 \%$ \\
2007 & $55,30 \%$ & $45,03 \%$ & $71,57 \%$ & $69,83 \%$ \\
\hline
\end{tabular}




\section{CONCLUSÕES}

O presente estudo representa a primeira aplicação em transporte aéreo a incorporar um terceiro estágio ao modelo NDEA. A análise compreende a avaliação do desempenho operacional das companhias aéreas brasileiras sob uma ótica diferente, que combina o modelo NDEA com a métrica SBM. As DMUs são as empresas aéreas regulares de transporte de carga e passageiros, considerando que a mesma companhia é uma unidade distinta em anos diferentes.

Das 69 DMUs analisadas, nove são globalmente eficientes. Três referem-se ao ano de 2010, duas a 2008 e apenas uma a 2007. O fato de o ano de 2007 apresentar o menor número de empresas globalmente eficientes sugere que a modelagem adotada de algum modo reflete a crise de 2006. Os resultados apontam, ainda, que sete DMUs ineficientes foram eficientes em ao menos um estágio, nenhuma delas no primeiro, indicando que a eficiência global parece estar fortemente ligada à eficiência no primeiro estágio, em especial às variáveis de frota e pessoal.

A utilização do modelo de NDEA permitiu a decomposição aditiva do processo produtivo em estágios interligados, facilitando a identificação das fontes de ineficiência ao longo da cadeia produtiva. Uma limitação dos modelos NDEA aditivos é que as informações fornecidas são insuficientes para determinar a fronteira eficiente.

Uma possível extensão deste trabalho seria incorporar os princípios de conjuntos difusos para lidar com a pouca precisão dos dados de frota, como feito em Correia et al. (2011), mas desta vez utilizando um modelo NDEA difuso (Lozano e Moreno, 2014). Pesquisas futuras incluem, ainda, a aplicação de modelos NDEA dinâmicos (Tone e Tsutsui, 2014), que incorporam o trânsito de atividades ao modelo NDEA e permitem a avaliação da eficiência de um período específico com base na otimização de longo prazo do período total.

\section{AGRADECIMENTOS}

Agradecemos à CNPq e à Fundación Carolina pelo apoio financeiro.

\section{REFERÊNCIAS BIBLIOGRÁFICAS}

[1] Adler, N.; Berechman, J. (2001). Measuring airport quality from the airlines' viewpoint: an application of data envelopment analysis. Transport Policy, 8(3), 171-181.

[2] Adler, N.; Golany, B.E. (2001). Evaluation of deregulated airline networks using data envelopment analysis combined with principal component analysis with an application to Western Europe, European Journal of Operational Research, 260-273.

[3] ANAC. Anuários estatísticos do transporte aéreo 2007. Disponível em: http://www.anac.gov.br/.

[4] ANAC. Anuários estatísticos do transporte aéreo $2008 . \quad$ Disponível em: http://www.anac.gov.br/.

[5] ANAC. Anuários estatísticos do transporte aéreo $2009 . \quad$ Disponível em: http://www.anac.gov.br/.

[6] ANAC. Anuários estatísticos do transporte aéreo 2010. Disponível em: http://www.anac.gov.br/.

[7] Araújo Junior, A.H.; Avellar, J.V.G.; Marins, F.A.S.; Milioni, A. Z. (2007). A eficiência operacional do transporte aéreo brasileiro. Engenharia, 582,122-130.

[8] Banker, R.D.; Charnes, A.; Cooper, W.W. (1984). Some models for estimating technical scale inefficiencies in data envelopment analysis. Management Science, 30(9), 1078-1092. 
[9] Banker, R.D.; Charnes, A.; Cooper, W.W.; Swarts, J.; Thomas, D. (1989). An introduction to data envelopment analysis with some models and their uses. Research in Governmental and Non-Profit Accounting, 5, 125-163.

[10] Barros, C.P.; Dieke, P.U.C. (2007). Performance evaluation of Italian airports: a data envelopment analysis. Journal of Air Transport Management, 13(4), 184-91.

[11] Capobianco, H.M.P.; Fernandes, E. (2004). Capital structure in the world airline industry. Transportation Research Part a-Policy and Practice, 38(6), 421-434.

[12] Chang, Y.-C.; Yu, M.-M. (2012). Measuring production and consumption efficiencies using the slack-based measure network data envelopment analysis approach: The case of low-cost carriers. Journal of Advanced Transportation.

[13] Chang, Y.-C; Yu, M.-M.; Chen, P.-C. (2013). Evaluating the performance of chinese airports. Journal of Air Transport Management, 31(1): 19-21.

[14] Charnes, A.; Cooper, W.W.; Rhodes, E. (1978). Measuring the efficiency of decisionmaking units. European Journal of Operational Research, 2, 429-444.

[15] Charnes, A.; Gallegos, A.; Li, H. (1996). Robustly efficient parametric frontiers via multiplicative DEA for domestic and international operations of the latin american airline industry. European Journal of Operational Research, 88(3), 525-536.

[16] Chen, Y.; Cook, W.D.; Li, N.; Zhu, J. (2009). Additive efficiency decomposition in two-stage DEA. European Journal of Operational Research, 196(3), 1170-1176.

[17] Chi-Lok, A.Y.; Zhang, A. (2009). Effects of competition and policy changes on chinese airport productivity: An empirical investigation. Journal of Air Transport Management, 15(4), 166-174.

[18] Correia, T.C.V.D.; Soares de Mello, J.C.C.B.; Angulo-Meza, L. (2011). Assessment of Brazilian airlines technical efficiency: A study using data envelopment analysis and fuzzy sets. Produção, 21(4), 676-683.

[19] Evangelho, F.; Huse, C.; Linhares, A. (2005). Market entry of a low cost airline and impacts on the Brazilian business travelers. Journal of Air Transport Management, 11 (2), 99-105.

[20] Fernandes, E.; Pires, H.M.; Lins, M.P.E.; Silva, A.C.M. (2008). Financial performance of air transport companies: An analysis of the non-pareto-efficient space in data envelopment analysis. Paper read at WIT Transactions on Information and Communication Technologies.

[21] Färe, R.; Grosskopf, S. (2000). Network DEA, Socio-Economic Planning Sciences, 34, 35-49.

[22] Fonseca, A.B.M.; Soares de Mello, J.C.C.B.; Gomes, E.G.; Angulo-Meza, L. (2010). Uniformization of frontiers in non-radial ZSG-DEA models: An application to airport revenues. Pesquisa Operacional, 30(1), 175-193.

[23] Gillen, D.; Lall, A. (1997). Developing measures of airport productivity and performance: an application of data envelopment analysis. Transportation Research Part E: Logistic and Transportation Review, 33(4), 261-73.

[24] Gomes Júnior, S.F.; Soares de Mello, J.C.C.B.; Angulo Meza, L. (2013). DEA nonradial efficiency based on vector properties, International Transactions in Operational Research, 20(3), 341-364. 
[25] Ha, H.K.; Wan, Y.; Yoshida, Y.; Zhang, A. (2013). Airline market structure and airport efficiency: Evidence from major Northeast Asian airports. Journal of Air Transport Management, 33, 32-42.

[26] Kao, C. (2009). Efficiency decomposition in network data envelopment analysis: A relational model. European Journal of Operational Research, 192(3), 949-962.

[27] Lima, V.S.; Soares de Mello, J.C.C.B.; Angulo-Meza, L. (2011). Cost-benefit analysis in selected air trips using a non-parametric method. African Journal of Business Management, 5(21), 9678-9685.

[28] Lozano, S. (2011). Scale and cost efficiency analysis of networks of processes. Expert Systems with Applications, 38 (6), 6612-6617.

[29] Lozano, S.; Gutiérrez, E.; Moreno, P. (2013). Network DEA approach to airports performance assessment considering undesirable outputs. Applied Mathematical Modelling, 37(4), 1665-1676.

[30] Lozano, S.; Moreno, P. (2014). Network fuzzy data envelopment analysis. Studies in Fuzziness and Soft Computing, 309, 207-230.

[31] Lu, W.-M.; Wang, W.-K.; Hung, S.-W.; Lu, E.-T. (2012).The effects of corporate governance on airline performance: Production and marketing efficiency perspectives. Transportation Research Part E: Logistics and Transportation Review, 48(2), 529-544.

[32] Pacheco, R.R.; Fernandes, E.; Santos, M.P.D. (2006). Management style and airport performance in Brazil. Journal of Air Transport Management, 12(6), 324-330.

[33] Podinovski, V.V.; Thanassoulis, E. (2007). Improving discrimination in data envelopment analysis: Some practical suggestions. Journal of Productivity Analysis, 28(1-2), 117-126.

[34] Sarkis, J. (2000). An analysis of the operational efficiency of major airports in the United States. Journal of Operations Management, 18(3), 335-351.

[35] Schefczyk, M. (1993). Operational performance of airlines: An extension of traditional measurement paradigms. Strategic Management Journal, 14, 301-317.

[36] Silveira, J.Q.; Soares de Mello, J.C.C.B.; Angulo-Meza, L. (2012a). Brazilian airlines efficiency evaluation using a data envelopment analysis (DEA) and multiobjective linear programming hybrid model. Ingeniare, 20(3), 331-342.

[37] Silveira, J. Q.; Soares de Mello; J.C.C.B.; Angulo-Meza, L. (2012b). Use of DEA and inverted frontier for airlines benchmarking and anti-benchmarking identification. Produção, 22(4), 788-795.

[38] Soares de Mello, J.C.C.B.; Angulo-Meza, L.; Gomes, E.G.; Serapião, B.P.; Lins, M.P.E. (2003). Análise de envoltória de dados no estudo da eficiência e dos benchmarks para companhias aéreas brasileiras. Pesquisa Operacional, 23(2), 325-345.

[39] Soares de Mello, J.C.C.B.; Angulo-Meza, L.; Silveira, J.Q.; Gomes, E.G. (2013). About negative efficiencies in cross evaluation bcc input oriented models. European Journal of Operacional Research, 229(3), 732-737.

[40] Soares de Mello, P.H.C.; Soares de Mello, J.C.C.B.; Angulo-Meza, L. (2009). Misunderstandings due to a codeshare between two Brazilian airlines in Rio de Janeiro international airport. Rio's International Journal on sciences of industrial and systems engineering and management, 3(1), 2. 
[41] Suzuki, S.; Nijkamp, P.; Pels, E.; Rietveld, P. (2012). Comparative performance analysis of european airports by means of extended data envelopment analysis. Journal of Advanced Transportation.

[42] Tone, K. (2001). A slacks-based measure of efficiency in data envelopment analysis. European journal of operational research, 130(3), 498-509.

[43] Tone, K.; Tsutsui, M. (2009). Network DEA: a slacks-based measure approach. European Journal of Operational Research, 197(1), 243-252.

[44] Tone, K.; Tsutsui, M. (2014). Dynamic DEA with network structure: A slacks-based measure approach. Omega (United Kingdom), 42(1), 124-131.

[45] Wanke, P.F. (2013) Physical infrastructure and flight consolidation efficiency drivers in Brazilian airports: A two-stage network-DEA approach. Journal of Air Transport Management, 31, 1-5.

[46] Yu, M.-M. (2010). Assessment of airport performance using the SBM-NDEA model. Omega, 38(6), 440-452.

[47] Yu, M.-M. (2012). Performance assessment of transport services with the ERM-NDEA model: Evidence from a domestic airline in Taiwan. Transportation Planning and Technology, 35(7), 697-714.

[48] Yu, M.-M.; Chen, P.-C. (2011). Measuring air routes performance using a fractional network data envelopment analysis model. Central European Journal of Operations Research, 19(1), 81-98.

[49] Zhu, J. (2011). Airlines Performance via Two-Stage Network DEA Approach. Journal of CENTRUM Cathedra, 4(2), 260-269. 